\title{
Nanoscale magnetic structure and properties of solution-derived self-assembled $\mathrm{La}_{0.7} \mathrm{Sr}_{0.3} \mathrm{MnO}_{3}$ islands
}

\author{
J. Zabaleta, ${ }^{1, a)}$ M. Jaafar, ${ }^{2}$ P. Abellán, ${ }^{1}$ C. Montón, ${ }^{1}$ O. Iglesias-Freire, ${ }^{2}$ F. Sandiumenge, ${ }^{1}$ \\ C. A. Ramos, ${ }^{3}$ R. D. Zysler, ${ }^{3}$ T. Puig, ${ }^{1}$ A. Asenjo, ${ }^{2}$ N. Mestres, ${ }^{1}$ and X. Obradors ${ }^{1}$ \\ ${ }_{1}^{1}$ Institut de Ciència de Materials de Barcelona ICMAB-CSIC, Campus de la UAB, Bellaterra 08193 Barcelona, \\ Spain \\ ${ }^{2}$ Instituto de Ciencia de Materiales de Madrid ICMM-CSIC, Sor Juana Inés de la Cruz 3, Cantoblanco 28049 \\ Madrid, Spain \\ ${ }^{3}$ Centro Atómico Bariloche and Instituto Balseiro, Comisión Nacional de Energía Atómica and Universidad \\ Nacional de Cuyo, South Carolina de Bariloche 8400, Argentina
}

(Received 4 September 2011; accepted 16 December 2011; published online 24 January 2012)

\begin{abstract}
Strain-induced self-assembled $\mathrm{La}_{0.7} \mathrm{Sr}_{0.3} \mathrm{MnO}_{3}$ nanoislands of lateral size $50-150 \mathrm{~nm}$ and height 10-40 nm have been grown on yttria-stabilized zirconia (001)-substrates from ultradiluted chemical solutions based on metal propionates. The nanoislands grow highly relaxed withstanding the epitaxial relation $(001)_{\text {LSMO }}[110] / /(001)_{\mathrm{YSZ}}[010]$ and show bulk-like average magnetic properties in terms of Curie temperature and saturation magnetization. The interplay of the magnetocrystalline and shape anisotropy within the nanoisland ensemble results in an in-plane magnetic anisotropy with a magnetocrystalline constant $K_{1}(150 \mathrm{~K})=-(5 \pm 1) \mathrm{kJ} / \mathrm{m}^{3}$ and in-plane easy axis along the [110] $-\mathrm{La}_{0.7} \mathrm{Sr}_{0.3} \mathrm{MnO}_{3}$ direction as measured, for the first time, through ferromagnetic resonance experiments. Magnetic force microscopy studies reveal the correlation between nanoisland size and its magnetic domain structure in agreement with micromagnetic simulations. In particular, we have established the required geometric conditions for single domain, multidomain, and vortex configurations. (C) 2012 American Institute of Physics. [doi:10.1063/1.3677985]
\end{abstract}

\section{INTRODUCTION}

Nanoscale magnetism has been object of intense research in the past years motivated by the new phenomena and by the potential applications that arise at reduced size and dimensionality. ${ }^{1,2}$ Moreover, the recent advances in nanofabrication and characterization techniques have led to a renewed interest in a wide variety of materials and to the discovery of new magnetic behaviors. ${ }^{3,4}$ The implementation of such nanomagnetic structures in devices with novel functionalities requires, nevertheless, controlling, and understanding the multiple factors influencing their magnetic properties.

A substantial amount of work exists on the patterning of magnetic metal thin films to submicron sizes, ${ }^{1,2,5,6}$ whereas fewer studies have been devoted to complex magnetic oxides. ${ }^{7,8}$ Among these, the mixed valence perovskite manganite $\mathrm{La}_{1-\mathrm{x}} \mathrm{Sr}_{\mathrm{x}} \mathrm{MnO}_{3}$ (LSMO), has triggered plenty of interest due to the physical phenomena that it exhibits, such as colossal magnetoresistance. ${ }^{9,10}$ In particular, LSMO with $\mathrm{Sr}$ doping of $x=0.3$ displays ferromagnetic and metallic behavior at room temperature and hence results especially appealing from an application point of view. In most cases, these LSMO nanostructures are attained by patterning continuous films into islands using optical and e-beam lithography or by combining e-beam lithography and ion milling. ${ }^{8,11,12}$ However, it is in general difficult to process oxides in such manner and to accomplish lateral sizes below $0.3 \mu \mathrm{m}$ without radiation damage or ion implantation. ${ }^{8}$ In this context, the bottom-up self-assembly approach based on the chemical solution deposition (CSD) route used in this

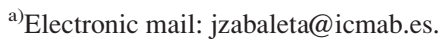

work offers an effective route to overcome the shortcomings of lithography-based techniques, while uniformly covering large areas (in the $\sim \mathrm{cm}^{2}$ range) without vacuum environment requirements. ${ }^{13-15}$

Inherent to heteroepitaxial growth, lattice strain may lead to relevant variations of the Curie temperature, electrical resistivity and magnetoresistance in nm thick films of mixedvalence manganite grown on bulk single crystals. ${ }^{16}$ Its effect is further evidenced on the magnetic anisotropy and microscopic magnetic domain configuration of, e.g., LSMO films and nanostructures. ${ }^{11,17,18}$ On the other hand, the magnetic properties of epitaxial strain-free nanometric LSMO nanoislands, where the shape and magnetocrystalline anisotropy are the key components, remain unexplored. The main reason for this is the aforementioned fabrication procedure, where nanostructures are attained from previously formed epitaxial thin films.

In this work we report on the novel system of selfassembled epitaxial $\mathrm{La}_{0.7} \mathrm{SrMnO}_{3}(\mathrm{LSMO})$ nanoislands grown through the CSD methodology onto (001)-oriented yttria stabilized zirconia (YSZ) substrates. In particular, we undertake the challenge of addressing the influence of key factors such as size and morphology, crystallographic structure, and strain, on the magnetic properties of our LSMO nanoislands. The morphological and structural characterization we first present is proved essential to be able to afterwards study the nanoisland magnetic anisotropy and deduce the magnetocrystalline anisotropy constant $K_{1}$. A Curie temperature $T_{C}$ comparable to bulk LSMO, remarkable in nanostructures with lateral sizes below $200 \mathrm{~nm}$, was measured using a superconducting quantum interference device (SQUID). Finally, local magnetic force microscopy (MFM) measurements reveal a rich 
landscape of magnetic structures, hidden behind the averaged magnetic behavior shown by the SQUID magnetization loops.

\section{EXPERIMENTAL DETAILS}

\section{A. Nanostructure growth}

$\mathrm{La}_{0.7} \mathrm{Sr}_{0.3} \mathrm{MnO}_{3}$ nanoislands were obtained following the CSD route from precursor solutions prepared by dissolving metal propionates in the stoichiometric ratio in propionic acid. ${ }^{19}$ Solution concentrations of $0.03 \mathrm{M}$ (with respect to the Mn cation) were used. The metalorganic solution was deposited on $5 \times 5 \mathrm{~mm}^{2}$ fluorite-structure (001)-YSZ single crystal substrates and spin-coated at $6000 \mathrm{rpm}$ for $2 \mathrm{~min}$. Finally, wet films were heated at high temperature $\left(900{ }^{\circ} \mathrm{C}-1000{ }^{\circ} \mathrm{C}\right)$ under oxygen atmosphere $\left(0.61 \mathrm{~min}^{-1}\right)$ for 1 to $5 \mathrm{~h}$. During this annealing process the organic matter decomposes and crystalline structures buildup leading to self-assembled nanoislands. We estimated the amount of crystallized LSMO material on the sample by distributing the totality of the island volume, calculated from atomic force microscopy (AFM) image analysis, into a hypothetical continuous film with a certain equivalent thickness. Such calculation gives an equivalent film thickness value that ranges from 3 to $4( \pm 0.5) \mathrm{nm}$.

The growth process above described yields the selfassembled LSMO nanoislands as a result of the interplay of the thermodynamics and kinetics of the system. We found a general tendency toward wider nanoisland size distributions and less homogeneous self-assemblies outside the ranges established in the previous lines. For instance, at lower temperatures and higher concentrations, respectively, crystallization was not optimal and coalescence processes yielded agglomerated islands. In addition to growth temperature and solution concentration, the distinct interface energies and lattice mismatches between LSMO and the chosen substrate also proved to be critical. We optimized the 3D growth on YSZ substrates $(-5.9 \%$ lattice mismatch of LSMO with respect to YSZ) after observing a 2D growth trend in initially tested perovskite-type substrates displaying lower mismatches.

\section{B. Morphological and structural characterization}

AFM was performed in the dynamic mode at room temperature and ambient atmosphere using an Agilent 5100 (Molecular Imaging) and a Cervantes system (Nanotec Electrónica S.L.). ${ }^{20} \mathrm{X}$-ray diffraction measurements were done with a GADDS D8 Advance system from Bruker with a 2D-detector. Transmission electron microscopy (TEM) images were obtained with a Jeol 2010 F electron microscope, while for electron diffraction pattern acquisition a Philips CM30 electron microscope was used, both of them operating at $200 \mathrm{kV}$. Cross-sectional samples for TEM examination were prepared using the mechanical tripod polisher technique followed by an $\mathrm{Ar}^{+}$milling step down to perforation in a Precision Ion Polishing System.

\section{Magnetic properties measurements}

Macroscopic magnetization measurements were made in a SQUID magnetometer (Quantum Design MPMS-XL7) at temperatures between 10 to $300 \mathrm{~K}$ and varying magnetic fields from 0 to $7 \mathrm{~T}$. Ferromagnetic resonance (FMR) measurements were performed in a Bruker ESP 300 spectrometer operated at $9.5 \mathrm{GHz}$ in a rectangular $\mathrm{x}$-band cavity operating in the TE102 mode. The FMR spectra reported here were done at $150 \mathrm{~K}$ keeping the applied field, $H$, in the sample plane. MFM imaging was performed with a Cervantes system (Nanotec Electrónica S.L.) working in noncontact mode and with the phase-locked loop (PLL) system activated. Experiments were carried out at room temperature and low humidity environment. A retrace distance of $\sim 30 \mathrm{~nm}$ was proved optimal for a good magnetic sensitivity while avoiding cross talk with the short-range topography signal. The probes were commercial Co-coated Nanosensors PPPMFMR and PPP-LM-MFMR cantilevers with $k=2.8 \mathrm{~N} / \mathrm{m}$ and $f=75 \mathrm{kHz}$. Although we observed slight changes in some nanoislands, in general, the stray field of the tips does not modify significantly the magnetic state of the sample. ${ }^{21}$ Micromagnetic simulations were performed using the object oriented micromagnetic framework 3D code (OOMMF eXtensible Solver $)^{22}$ with cell sizes of $2 \mathrm{~nm} \times 2 \mathrm{~nm} \times 1 \mathrm{~nm}$.

\section{RESULTS AND DISCUSSION}

LSMO nanoislands were grown using the CSD methodology described above. Figure 1 shows the morphological characterization of the system. We there observe how the growth of LSMO, with a perovskite crystallographic structure, on top of YSZ, with a highly dissimilar fluorite structure, results in the Volmer Weber growth mechanism with island formation. The island self-assembly takes place homogeneously on the whole substrate surface $\left(\sim 5 \times 5 \mathrm{~mm}^{2}\right)$ with a $\sim 20 \%$ of area coverage. From a great number of scans and measured nanoislands they were seen to exhibit thickness $t$ between 10-40 $\mathrm{nm}$ and lateral size $D$ between 50 and $150 \mathrm{~nm}$. Notice also the truncated square pyramid geometry shown in the TEM crosssectional view of Fig. 1(b). Taking the aspect ratio $D / t$, we obtain the statistical distribution shown in Fig. 1(c), with a mean value for $D / t$ of 4 and a standard deviation of 1 . These results are fully representative of every sample processed under the conditions specified in the experimental section, proving the robustness of the CSD methodology in the generation of self-assembled LSMO nanoislands.

The crystallographic orientation of the LSMO nanoislands on YSZ was measured by x-ray diffraction (XRD) $\theta-2 \theta$ profiles and pole figures (Fig. 2). The area covered by the X-rays $\left(<1 \mathrm{~mm}^{2}\right)$ along with the standard sensitivity of the measurements (typically $2-3$ wt. \%) was proved sufficient to obtain signal from the LSMO nanoisland ensemble. $\theta-20 \mathrm{x}$-ray diffraction scans of the LSMO nanoislands on (011) - YSZ reveal the presence of the LSMO $\{011\}$ peak family (in terms of the pseudocubic lattice, ${ }^{23} a_{\text {LSMO }}=3.78 \AA$ ) [see Fig. 2(a)]. Figure 2(b) shows the pole figures corresponding to the (011) LSMO and (022) YSZ reflections. In both cases the poles appear at $\chi=45^{\circ}$ [consistent with the (001) orientation], but are rotated $\phi=45^{\circ}$ to each other, indicating the epitaxial relationship (001) LSMO $_{\text {LS }}[10] / /(001)_{\mathrm{YSZ}}[010]$ Hence, the diffraction data prove that the vast majority of the LSMO nanoislands grow epitaxially on top of the YSZ substrate with a single crystalline orientation. The sketched diagram in Fig. 2(b) highlights 

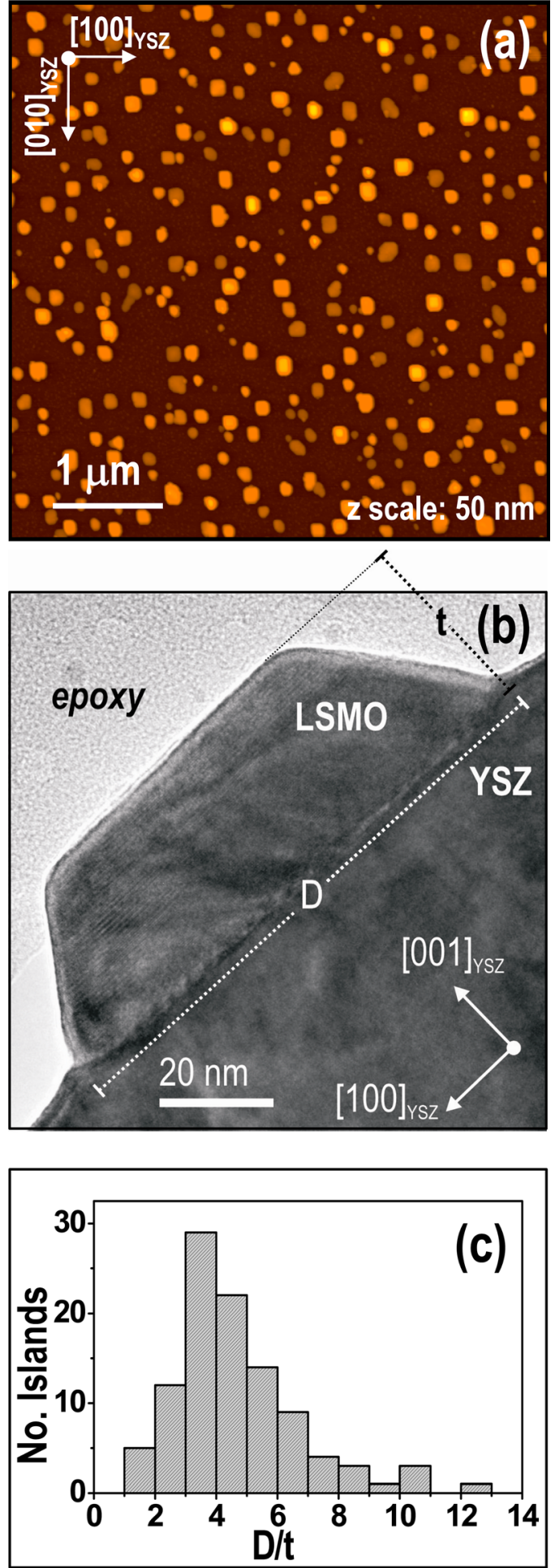

FIG. 1. (Color online) (a) $5 \times 5 \mu \mathrm{m}^{2}$ AFM topography image of LSMO nanoislands grown at $900{ }^{\circ} \mathrm{C}$ during $1 \mathrm{~h}$ on (001)-YSZ. (b) Cross-sectional TEM image of an LSMO nanoisland with aspect ratio $D / t \sim 3.5$, displaying the typical truncated pyramid geometry. (c) Distribution of the $D / t$ values displayed by the nanoisland ensemble.

the nanoisland epitaxial relationship with respect to the YSZ substrate, along with its square-pyramid nanoisland morphology, with (001) LSMO top and (111) LSMO lateral facets. The LSMO[110]//YSZ[010] interface arrangement would correspond to a nominal misfit $\varepsilon \sim-5.9 \%$ between the [110] direction of $\operatorname{LSMO}\left(\sqrt{ } 2 a_{\mathrm{LSMO}}=0.547 \mathrm{~nm}\right)$ and the [010]
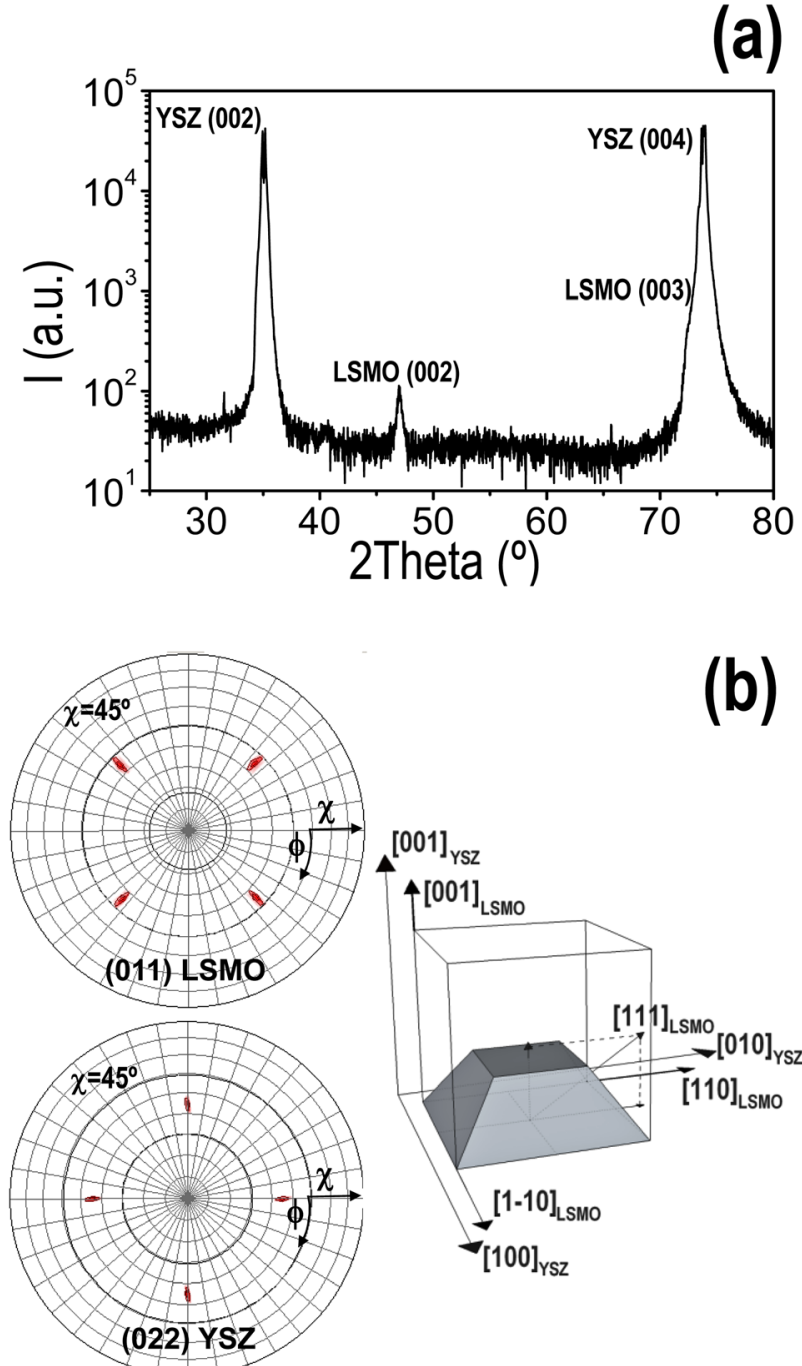

FIG. 2. (Color online) (a) X-ray $\theta-2 \theta$ diffractogram of LSMO nanoislands grown at $900{ }^{\circ} \mathrm{C}$ for $1 \mathrm{~h}$ on (001)-YSZ. (b) X-ray diffraction pole figures of the (011) LSMO reflection and of the (022) YSZ reflection corresponding to $(001)_{\text {LSMO }}[110] / /(001)_{\text {YSZ }}[010]$ oriented nanoislands. The epitaxial relationship and the island square-pyramid shape are sketched on the right.

direction of YSZ $\left(a_{\mathrm{YSZ}}=0.515 \mathrm{~nm}\right)$. This significant difference in lattice mismatch, which initially suggests a highly strained heteroepitaxy, may however translate in an unfavorably large building up of strain energy, which could, in turn, lead to the relaxation of the nanostructure. As a matter of fact, the LSMO lattice parameter deduced from the $\theta-2 \theta$ scans corresponds to the bulk LSMO value, indicating a highly relaxed structure.

Further evidence of the nanoisland strain-state was obtained using high resolution TEM and electron diffraction (ED) measurements. Figure 3(a) is an ED pattern of a selected area simultaneously containing the YSZ substrate and a single LSMO nanoisland. The epitaxial relationship of the individual island with respect to the YSZ substrate is in agreement with the XRD measurements, obtained for a large nanoisland ensemble. Notice also that the sublattices from substrate and nanoisland can be easily distinguished with the naked eye. Furthermore, a measure of the lattice spacing separation is consistent with a complete relaxation of the lattice parameters 
for LSMO. A high resolution TEM (HRTEM) image of an interfacial region is shown in Fig. 3(b). Fourier filtering analyses revealed that the strain contrast observed at the interface corresponds to dislocations with Burgers vector $b=\left(a_{\mathrm{YSZ}} / 2\right)[100]$ (arrowed in the image), with the extra half plane residing in the substrate in order to accommodate the compressive lattice misfit in the island. The average distance measured in the image, $\sim 4.0 \mathrm{~nm}$, coincides with the theoretical value $|b| / \varepsilon$ for a complete misfit relaxation. Our results thus confirm that the LSMO islands on YSZ are free of misfit strains.

Once the morphology, crystal structure and strain-state of our nanoislands are known, we move on to investigate their magnetic properties. The temperature dependence of the spontaneous magnetization of the LSMO nanoisland ensemble, measured in a SQUID magnetometer at an applied field of $50 \mathrm{mT}$, is shown in Fig. 4(a). Note that a Curie temperature $T_{C} \sim 350 \pm 3 \mathrm{~K}$ close to that of the bulk $\mathrm{LSMO}^{9}$ is attained. This high $T_{C}$ value can be correlated to the absence of strain within the islands-deduced from XRD and TEM investigations-and also linked to the noninvasive self-assembly growth process, which precludes magnetic properties to be depressed from ion implantation or radiation damage. Figure 4(b) displays the magnetization curves measured at $110 \mathrm{~K}$ with in-plane and out-of-plane applied field and reveals that the easy magnetization axis lies within the sample plane. However, because of the small amount of material and thus the low magnetic signal, it was not possible to discern with
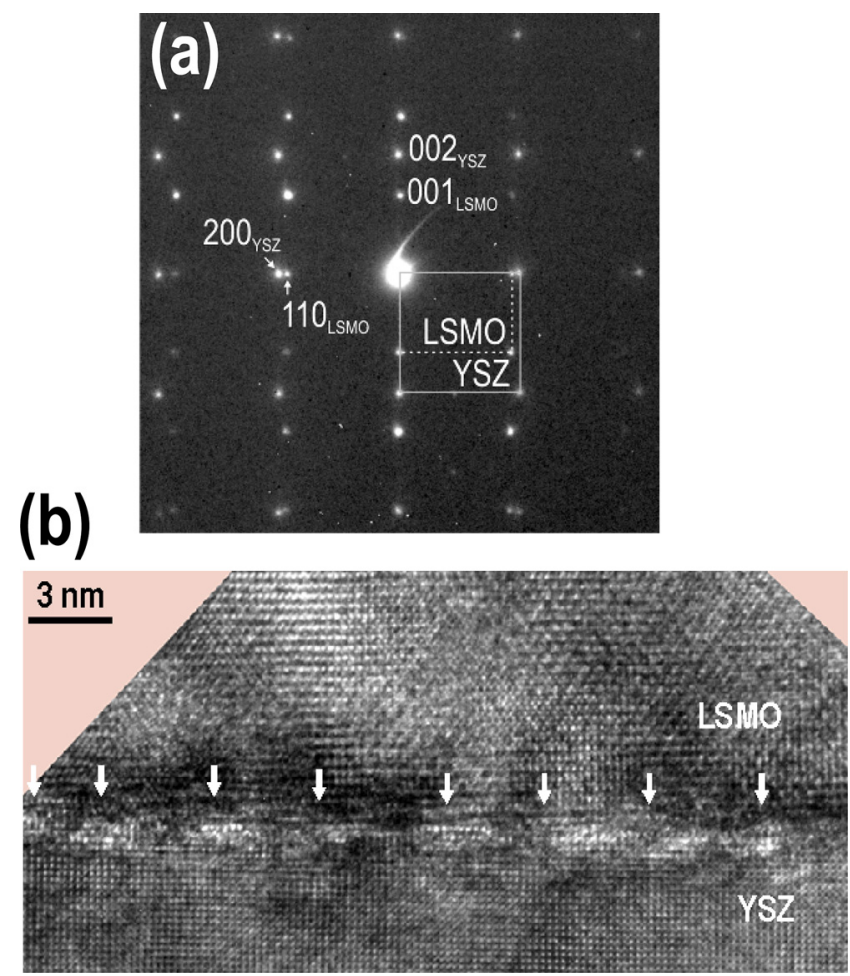

FIG. 3. (Color online) (a) ED pattern taken across a LSMO island-substrate interface showing the epitaxial relationship $(001)_{\text {LSMO }}[110] / /(001)_{\text {YSZ }}[010]$. The LSMO and YSZ lattices are marked by dashed and continuous lines, respectively. (b) Cross-sectional HRTEM image of a (001)-oriented LSMO nanoisland, where the arrows indicate regularly spaced misfit dislocations at the island/substrate interface. The LSMO island and the YSZ substrate are viewed along the [110] and [010] zone axes, respectively.
SQUID measurements which of the in-plane orientations corresponds to the easy magnetization direction. Coercive fields of $\sim 15 \mathrm{mT}$ and a saturation magnetization value $M_{s}=(480 \pm 50) \mathrm{kA} / \mathrm{m}($ at $35 \mathrm{~K})$, somewhat below the value for bulk LSMO $(590 \mathrm{kA} / \mathrm{m})$, were also obtained. The large error value in $M_{\mathrm{s}}$ stems from the uncertainty in the total LSMO volume estimation (see Sec. II). Because of this uncertainty we cannot rule out the presence of a ferromagnetic dead

(a)
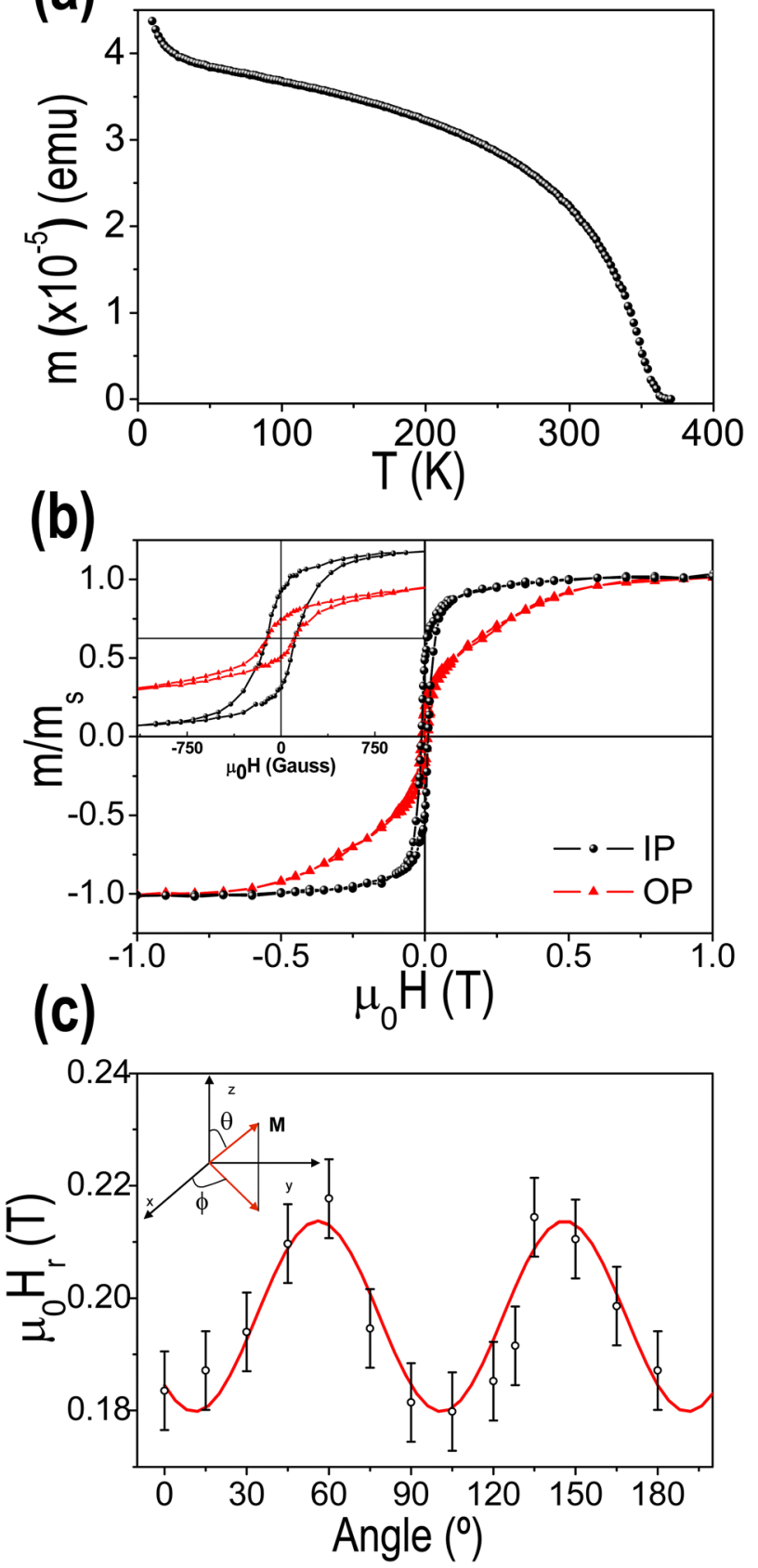

FIG. 4. (Color online) (a) Temperature dependence of the magnetization measured at $50 \mathrm{mT}$ displaying a $T_{C} \sim 350 \mathrm{~K}$ for the system of selfassembled LSMO nanoislands on (001)-YSZ. (b) The measured in-plane (IP) and out-of-plane (OP) magnetic hysteresis loops (110 K) evidence the in-plane anisotropy of the system. (c) Results from the ferromagnetic resonance measurements. Open symbols represent the measured in-plane dependence of the resonant field $H_{r}$ at $150 \mathrm{~K}$; the solid line is the fitting to the experimental data according to Eq. (3); the inset shows the coordinate system used. 
layer $(\sim 1-2 \mathrm{~nm}$ ) which has been subject of intense study and no little controversy in manganite based systems. ${ }^{24}$

From the factors that could influence the magnetic anisotropy of the nanoislands, i.e., the compressive strain that could pull the magnetization out-of-plane, their shape, and the magnetocrystalline anisotropy, the first does not play a role because of the relaxed nanoisland state, while the flat island shape $(D / t \sim 4)$ appears to favor the in-plane magnetization. To reveal the role of the magnetocrystalline anisotropy, we carefully studied the nanoisland ensemble by means of Ferromagnetic Resonance (FMR). Figure 4(c) (open circles) shows the measured in-plane angular dependence of the resonant field $H_{r}$. This value is obtained at each angle by fitting the FMR spectra with a Lorentzian line shape. Experimental data show a clear angular dependence of $H_{r}$ at $150 \mathrm{~K}$. Note that a minimum in $H_{r}$ indicates an easy magnetization axis, in this case the [110] axis of the LSMO nanoislands. To obtain the cubic magnetic anisotropy constant $K_{1}$ we followed a standard formalism. ${ }^{25}$ The energy density function is defined as follows, developing up to the first order the magnetocrystalline anisotropy term:

$$
\begin{aligned}
E= & -\mu_{0} \vec{H} \cdot \vec{M}+\frac{\mu_{0}}{2} M^{2}\left(N_{x} \alpha_{x}^{2}+N_{y} \alpha_{y}^{2}+N_{z} \alpha_{z}^{2}\right) \\
& +K_{1}\left(\alpha_{x}^{2} \alpha_{y}^{2}+\alpha_{x}^{2} \alpha_{z}^{2}+\alpha_{y}^{2} \alpha_{z}^{2}\right)
\end{aligned}
$$

where the first term represents the Zeeman energy, the second is the magnetostatic contribution $\left(N_{\mathrm{x}}, N_{\mathrm{y}}, N_{\mathrm{z}}\right.$ are the demagnetizing factors) and the third is the cubic crystalline anisotropy with $\alpha_{i}$ the direction cosines of the magnetization $\vec{M}$. In this in-plane configuration, no magnetoelastic contribution should be expected when the strain exerted by the substrate on the pseudocubic LSMO is isotropic. ${ }^{26}$ Moreover, we can rule out any possible deviation from a truly isotropic in-plane strain, since nanoislands are fully relaxed. Concerning the magnetostatic contribution, we estimated the demagnetizing field of a flat square-based pyramid magnetized perpendicularly to its base and found $N_{\perp}=0.70 \pm 0.05$ for $D / t$ in the range of $4 \pm 1$. Considering $N_{X}+N_{Y}+N_{\perp}=1\left(N_{X}=N_{Y}=N_{\|} \approx 0.15\right)$ it turns out that the effective shape demagnetizing factor for the applied field, $H$, parallel to the pyramid base, depends only on the difference $\left(N_{\perp}-N_{\|}\right)=\Delta N$. We rewrite Eq. (1) using the coordinate systems sketched in the inset of Fig. 4(c):

$$
\begin{aligned}
E= & -\mu_{0} M H\left[\sin \theta \cos \left(\phi-\phi_{H}\right)\right]+\frac{\mu_{0}}{2} M^{2} \Delta N \cos ^{2} \theta \\
& +\frac{K_{1}}{4}\left(\sin ^{4} \theta \sin ^{2} 2 \phi+\sin ^{2} 2 \theta\right),
\end{aligned}
$$

where $(\theta, \phi)$ define the position of $\vec{M}$ [Fig. 4(c)] and $\vec{H}$ is applied in the $x y$ plane at an angle $\phi_{H}$ with respect to the [100] axis. From the conditions $d E / d \theta=0$ and $d E / d \phi=0$ we reach the corresponding equilibrium positions $\theta=\pi / 2$ and, by assuming $K_{1} / M \ll \mu_{0} H, \phi=\phi_{H}$, thus indicating that $\vec{M}$ lies in the $x y$ plane and that it is parallel to the applied field. Evaluating the second derivative of Eq. (2) at the equilibrium positions $\theta=\pi / 2$ and $\phi=\phi_{H}$ and replacing the result in the Smit-Beljers equation, ${ }^{25}$ we obtain the FMR condition for our system of nanoislands

$$
\begin{aligned}
\left(\frac{\omega}{\gamma}\right)^{2}= & {\left[\mu_{0} H+\mu_{0} \Delta N M+\frac{2 K_{1}}{M}\left(1-\frac{1}{2} \sin ^{2} 2 \phi\right)\right] } \\
& \times\left(\mu_{0} H+\frac{2 K_{1}}{M} \cos 4 \phi\right)
\end{aligned}
$$

where $\omega / \gamma=0.34 \mathrm{~T}, \omega=2 \pi \nu$ with $\nu=9.5 \mathrm{GHz}$ the $\mathrm{x}$-band microwave frequency used and $\gamma$ the gyromagnetic factor for LSMO. The fitting of the experimental data to the Eq. (3) [solid line in Fig. 4(c)] yields the values $\mu_{0} \Delta N M(150 \mathrm{~K})=(0.393 \pm 0.004) \mathrm{T}$ and $\left(2 K_{1} / M\right)(150 \mathrm{~K})$ $=-(0.021 \pm 0.002) \mathrm{T}$.

Our numerical estimation of the flat pyramids' effective demagnetizing field give $\mu_{0} \Delta N M(150 \mathrm{~K})=(0.3 \pm 0.1) \mathrm{T}$, where we have introduced the measured saturation magnetization at $150 \mathrm{~K} M=0.9 \times(480 \pm 50) \mathrm{kA} / \mathrm{m}$, reduced a $10 \%$ from the low temperature value. Thus both values compare well and prove the validity of the assumptions made in the treatment. On the other hand, the value for the magnetocrystalline anisotropy constant $K_{1}$ is deduced from $2 K_{1} / M$ giving $K_{1}(150 K)=-(5 \pm 1) \mathrm{kJ} / \mathrm{m}^{3}$. This result is in the order of what it is reported in literature for LSMO films grown along the (001) plane which show the [110] in-plane easy axis. ${ }^{17,27}$ To our knowledge, this is the first reported shape anisotropy study for LSMO nanoislands along with the first measurement of $K_{l}$ in such nanoscale systems.

Upon the macroscopic analysis of the ferromagnetism of the nanoisland ensemble, we found of great interest to try to unveil their individual nanoscale magnetic structure. The tuning of the measuring conditions was essential to be able to image the magnetic response of such small nanostructures. Figure 5 displays the topography and corresponding magnetic force images of the surface, taken after saturating the tip and the sample in opposite directions by applying an out-of-plane magnetic field of $500 \mathrm{mT}$. Bright and dark MFM contrasts correspond to repulsive and attractive interactions, respectively. We may observe three different magnetic configurations as a function of the thickness $t$ and the lateral size $D$ of the nanoislands ( $D$ being the average between the two inplane values): low contrast, vortex state, and multidomain configurations. The average lateral size and thickness can be used to calculate the average section $S_{a}$ of every nanoisland configuration. Notice that due to the magnetic coating of the MFM tips, the radio of the probes increases and the quality of the topographic images decreases. The convolution between the tip and the sample was taken into account to evaluate the lateral dimensions of the islands. The magnetic configuration of the smaller islands $\left(S_{a} \sim 0.75 \times 10^{3} \mathrm{~nm}^{2}\right)$ cannot be determined due to the small interaction with the MFM tip. The medium size nanostructures $\left(S_{a} \sim 1.33 \times 10^{3} \mathrm{~nm}^{2}\right)$ present inplane anisotropy with vortex state, showing the characteristic out-of-plane magnetic contrast in the center of the nanoisland. Finally, the larger structures $\left(S_{a} \sim 2.50 \times 10^{3} \mathrm{~nm}^{2}\right)$ present multidomain magnetic configuration. The nonzero outof-plane components of the magnetic moment within the larger islands agrees with the macroscopic hysteresis loops measured in the out-of-plane configuration, which, although small, still show remanence magnetization and non negligible coercive field values [Fig. 4(b)]. 
(a)

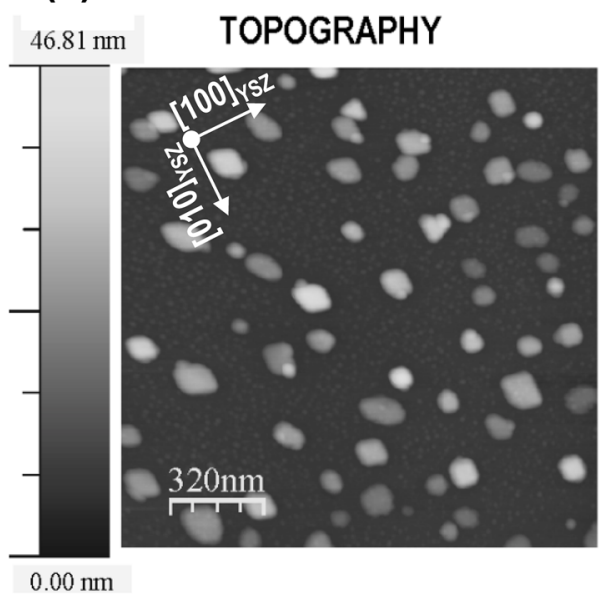

(b)

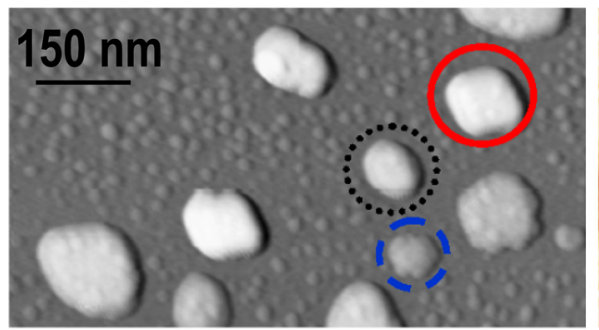
$2.8 \mathrm{~Hz}$

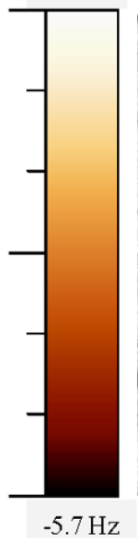

$-5.7 \mathrm{~Hz}$

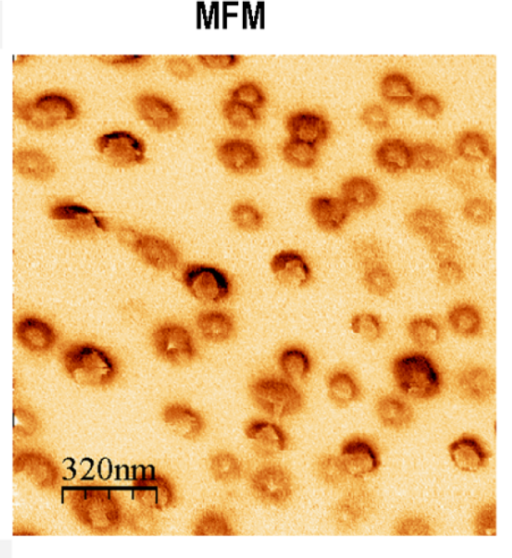

FIG. 5. (Color online) (a) Topographic (AFM) and magnetic (MFM) images of LSMO nanoislands at room temperature taken from the same location (scan size $1.6 \times 1.6 \mu \mathrm{m}^{2}$ ). (b) Detailed topographic and magnetic images of a smaller region of LSMO nanoislands. The dashed line, dotted line and solid line circles highlight nanoislands with single domain, vortex, and multidomain magnetic configurations, respectively.

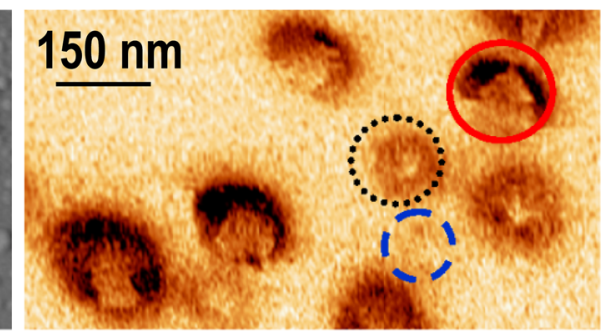

Statistical processing of the obtained MFM images shows that the above mentioned configurations fall into three distinct regions in the graph of Fig. 6(a). In other words, there exists a clear correlation between the geometrical parameters of the nanoisland and its magnetic ground state. In particular, nanoislands with $t$ of the order of the exchange length and with a lateral size below $110 \mathrm{~nm}$ display a vortex state, in good agreement with previous observations in magnetic metal nanostructures. ${ }^{6,28,29}$ Nanoislands with multidomain contrast correspond to those with larger $D$ and $t$ values, while the smaller nanoislands present a low contrast that seemingly corresponds to a homogeneous in-plane magnetization. Assuming a dead layer of thickness $\sim 2 \mathrm{~nm}$, the ferromagnetic section of this low contrast islands would be $8 \mathrm{~nm}$ thick [the average $t$ for these islands is $\sim 10 \mathrm{~nm}$, see Fig. 6(a)]. Such nanoislands can be divided into elements $8 \mathrm{~nm}$ in height that interact with the MFM tip. The force gradient between each element and the tip is about $3.8 \times 10^{-5} \mathrm{~N} / \mathrm{m}^{30}$ The total magnetic interaction between the Co coated MFM tip and a LSMO island has been estimated as the summation of the interactions between each element and the Co tip, giving $\sim 8.0 \times 10^{-4} \mathrm{~N} / \mathrm{m}$. However, this estimation is valid for outof-plane magnetized low contrast islands, and is one order of magnitude above the measured experimental average MFM value $8 \times 10^{-5} \mathrm{~N} / \mathrm{m}$. Such difference could be well explained if nanoislands actually displayed an in-plane magnetization. Note also that the experimental value is a factor two higher than the MFM signal noise for this particular kind of tip (evaluated as the MFM signal fluctuation out of the islands). This points out that the contrast of the so-called low contrast nanoislands is in principle measurable with our MFM system, however it is also at the limit of detection. Anyhow, the observed diversity in the magnetic configuration cannot be inferred from the SQUID hysteresis loops. In such macroscopic measurements the sizedependent magnetic behaviors all add up and reflect the average magnetic characteristics of the nanoisland ensemble.

To confirm the feasibility of our MFM results interpretation, we simulated the stable magnetization configurations using micromagnetic elements, ${ }^{22}$ taking a rectangular prism shape in first approximation. We introduced the experimental saturation magnetization and magnetocrystalline anisotropy constant values, and the exchange stiffness $A=1.73$ $\times 10^{-12} \mathrm{~J} / \mathrm{m}$ found in the literature. ${ }^{31}$ Representative island sizes of each of the three magnetic states were considered: small $(D \sim 50 \mathrm{~nm}, t \sim 10 \mathrm{~nm})$, medium-size $(D \sim 100 \mathrm{~nm}$, $t \sim 15 \mathrm{~nm})$, and large $(D \sim 120 \mathrm{~nm}, t \sim 30 \mathrm{~nm})$, nanoislands. The simulation results reveal three different magnetic states consistent with the experimental observations: small islands [Fig. 6(b)] exhibit a single domain configuration with in-plane magnetization (in agreement with the measured signal, lower than the one estimated, as previously explained). Large islands show a complex magnetization configuration [Fig. 6(d)], with out-of-plane magnetization components in different regions of the island. Feasibly, by using more realistic modeling of the larger islands, a better agreement between the magnetic configuration and the simulation could be reached. In between, the counterbalance of magnetostatic, exchange, and magnetocrystalline contributions results in the in-plane curling of the magnetic moment with an out-of-plane singularity at the center: i.e., the so-called vortex state. A vortex, displaying four independent and energetically equivalent states has become an appealing memory unit candidate for data 
(a)

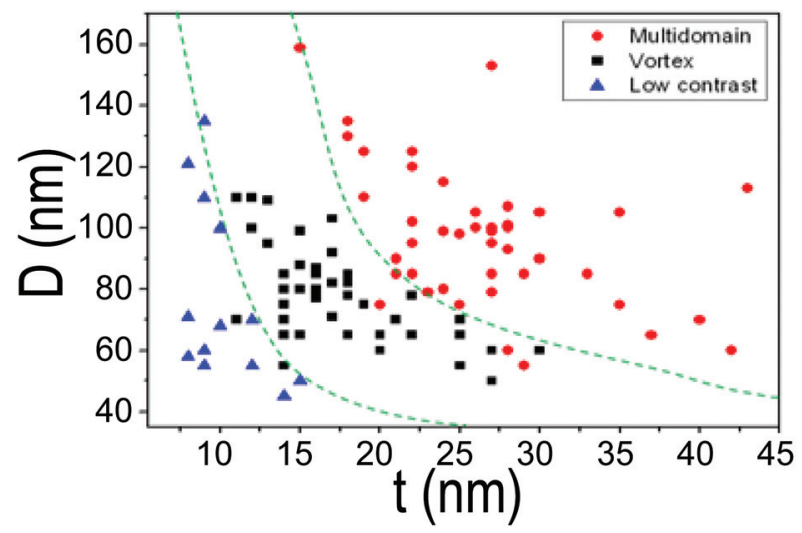

(b) (c) (d)

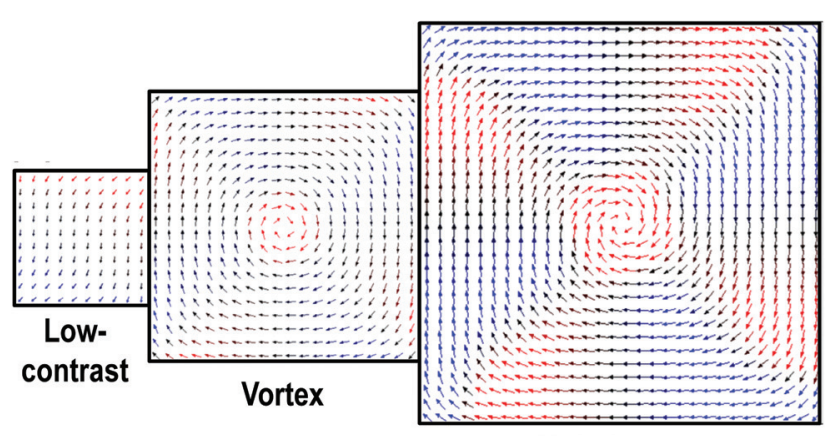

Multidomain

FIG. 6. (Color) (a) Phase diagram extracted from MFM measurements showing the magnetic configuration of the self-assembled LSMO nanoislands as a function of nanoisland dimensions (dashed lines represent a guide to the eye). Micromagnetic simulation results for (b) small (c) medium-size and (d) large nanoislands. The data scale for all the simulated images is $25 \mathrm{kA} / \mathrm{m}$. The blue and red colors correspond to the out of plane magnetization (outwards and inwards, respectively). The magnetization configurations correspond to the islands after saturating along the $+Z$ (outwards) direction.

storage. ${ }^{32,33}$ To be able to confine the geometries at which the vortex state is stable in LSMO nanoislands is hence of great interest toward potential data storage applications.

\section{CONCLUSIONS}

We have presented a comprehensive study of the structural and magnetic characteristics of a homogeneous system of ferromagnetic LSMO nanoislands grown from chemical methods. This system features highly crystalline, epitaxial and strain-relaxed nanoislands, below $200 \mathrm{~nm}$ in lateral size, and with a macroscopic $T_{c}$ comparable to bulk LSMO. Furthermore, we have calculated the magnetocrystalline anisotropy constant $K_{1}(150 \mathrm{~K})=-(5 \pm 1) \mathrm{kJ} / \mathrm{m}^{3}$ of the nanoisland ensemble, which is seen to display a biaxial in-plane anisotropy with the $[110]_{\text {LSMO }}$ in-plane easy axis. Insight into the local magnetic structure was given by MFM measurements and micromagnetic simulations. The MFM experiments reveal the existence of three size-dependent magnetic structures, and provide for the geometry required to stabilize the vortex state in LSMO nanostructures at room temperature.

\section{ACKNOWLEDGMENTS}

We acknowledge the financial support from MEC (MAT2008-01022, Consolider NANOSELECT and FPU), Comunidad Autónoma de Madrid (CAM S2009/MAT-1467), Generalitat de Catalunya (Catalan Pla de Recerca 2009-SGR770 and XaRMAE), and EU (NESPA). R. D. Zysler and C. A. Ramos acknowledge support from PIP-1333(2007) CONICET and PICT 829 (2006) and PICT 832(2006) ANPCyT of Argentina. Serveis Científic-Tècnics from Universitat de Barcelona and Servei de Micròscopia from Universitat Autònoma de Barcelona are acknowledged for TEM facilities.

${ }^{1}$ M. Hehn, K. Ounadjela, J. P. Bucher, F. Rousseaux, D. Decanini, B. Bartenlian, and C. Chappert, Science 272, 1782 (1996).

${ }^{2}$ J. I. Martín, J. Nogués, K. Liu, J. L. Vicent, and I. K. Schuller, J. Magn. Magn. Mater. 256, 449 (2003).

${ }^{3}$ R. Beaulac, L. Schneider, P. I. Archer, G. Bacher, and D. R. Gamelin, Science 325, 973 (2009).
${ }^{4}$ X. S. Gao, L. F. Liu, B. Birajdar, M. Ziese, W. Lee, M. Alexe, and D. Hesse, Adv. Funct. Mater. 19, 3450 (2009).

${ }^{5}$ C. A. Ross, R. Chantrell, M. Hwang, M. Farhoud, T. A. Savas, Y. Hao, H. I. Smith, F. M. Ross, M. Redjdal, and F. B. Humphrey, Phys. Rev. B 62, $14252(2000)$.

${ }^{6}$ T. Shinjo, T. Okuno, R. Hassdorf, K. Shigeto, and T. Ono, Science 289, 930 (2000).

${ }^{7}$ E. Folven, T. Tybell, A. Scholl, A. Young, S. T. Retterer, Y. Takamura, and J. K. Grepstad, Nano Lett. 10, 4578.

${ }^{8}$ Y. Takamura, R. V. Chopdekar, A. Scholl, A. Doran, J. A. Liddle, B. Harteneck, and Y. Suzuki, Nano Lett. 6, 1287 (2006).

${ }^{9}$ J. M. D. Coey, M. Viret, and S. von Molnar, Adv. Phys. 48, 167 (1999).

${ }^{10}$ A. Urushibara, Y. Moritomo, T. Arima, A. Asamitsu, G. Kido, and Y. Tokura, Phys. Rev. B 51, 14103 (1995).

${ }^{11}$ M. Mathews, R. Jansen, G. Rijnders, J. C. Lodder, and D. H. A. Blank, Phys. Rev. B 80, 064408 (2009).

${ }^{12}$ E. J. Kim, J. L. R. Watts, B. Harteneck, A. Scholl, A. Young, A. Doran, and S.Yuri, J. Appl. Phys. 109, 07D712 (2011).

${ }^{13}$ M. Gibert, T. Puig, X. Obradors, A. Benedetti, F. Sandiumenge, and R. Huhne, Adv. Mater. 19, 3937 (2007).

${ }^{14}$ C. Moreno, P. Abellán, A. Hassini, A. Ruyter, A. Pérez del Pino, F. Sandiumenge, M. J. Casanove, J. Santiso, T. Puig, and X. Obradors, Adv. Funct. Mater. 19, 2139 (2009).

${ }^{15}$ J. Zabaleta, N. Mestres, P. Abellan, M. Gibert, F. Sandiumenge, T. Puig, and X. Obradors, Nanotechnology 21, 025302 (2010).

${ }^{16}$ C. Adamo, X. Ke, H. Q. Wang, H. L. Xin, T. Heeg, M. E. Hawley, W. Zander, J. Schubert, P. Schiffer, D. A. Muller, L. Maritato, and D. G. Schlom, Appl. Phys. Lett. 95, 112504 (2009).

${ }^{17}$ K. Steenbeck and R. Hiergeist, Appl. Phys. Lett. 75, 1778 (1999).

${ }^{18}$ Y. Suzuki, H. Y. Hwang, S. W. Cheong, T. Siegrist, R. B. van Dover, A. Asamitsu, and Y. Tokura, J. Appl. Phys. 83, 7064 (1998).

${ }^{19}$ U. Hasenkox, C. Mitze, R. Waser, R. R. Arons, J. Pommer, and G. Guntherodt, J. Electroceram. 3, 255 (1999).

${ }^{20}$ I. Horcas, R. Fernandez, J. M. Gomez-Rodriguez, J. Colchero, J. GomezHerrero, and A. M. Baro, Rev. Sci. Instrum. 78, 013705 (2007).

${ }^{21}$ M. Jaafar, A. Asenjo, and M. Vazquez, IEEE Trans. Nanotechnol. 7, 245 (2008).

${ }^{22}$ See http://math.nist.gov/oommf/ for more information on the micromagnetic simulations.

${ }^{23}$ M. C. Martin, G. Shirane, Y. Moritomo, and Y. Tokura, Phys. Rev. B 53, 14285 (1996).

${ }^{24}$ J. Curiale, M. Granada, H. E. Troiani, R. D. Sanchez, A. G. Leyva, P. Levy, and K. Samwer, Appl. Phys. Lett. 95, 043106 (2009).

${ }^{25}$ J. Smit and H. G. Beljers, Philips Res. Rep. 10, 113 (1955).

${ }^{26}$ S. Chikazumi, Physics of Magnetism (Wiley, New York, 1964).

${ }^{27}$ K. Steenbeck, T. Habisreuther, C. Dubourdieu, and J. P. Senateur, Appl. Phys. Lett. 80, 3361 (2002).

${ }^{28}$ R. P. Cowburn, D. K. Koltsov, A. O. Adeyeye, M. E. Welland, and D. M. Tricker, Phys. Rev. Lett. 83, 1042 (1999). 
${ }^{29}$ A. Wachowiak, J. Wiebe, M. Bode, O. Pietzsch, M. Morgenstern, and R. Wiesendanger, Science 298, 577 (2002).

${ }^{30} \mathrm{We}$ have estimated the interaction between a Co coated tip [radius $\sim 10$ $\mathrm{nm}$ and $\left.M_{S}(\mathrm{Co})=1440 \mathrm{kA} / \mathrm{m}\right]$ and a LSMO nanoisland divided into $8 \mathrm{~nm}$ size elements with $M_{s}(\mathrm{LSMO}$ at $300 \mathrm{~K})=240 \mathrm{kA} / \mathrm{m}$, using $\partial F / \partial z=\sum_{i}$ $\left(\mu_{0} 6 m_{\text {tip }} m_{i}\right) /(\pi z)$, where $m_{\text {tip }}$ and $m_{i}$ are the magnetic moment of the tip and the magnetic moment of one of the elements of the nanoisland, respectively, and $z$ is the distance between the tip and each nanoelement.
${ }^{31}$ R. Akiyama, H. Tanaka, T. Matsumoto, and T. Kawai, Appl. Phys. Lett. 79, 4378 (2001)

${ }^{32}$ B. Pigeau, G. De Loubens, O. Klein, A. Riegler, F. Lochner, G. Schmidt, L. W. Molenkamp, V. S. Tiberkevich, and A. N. Slavin, Appl. Phys. Lett. 96, 132506 (2010).

${ }^{33}$ A. Ruotolo, V. Cros, B. Georges, A. Dussaux, J. Grollier, C. Deranlot, R. Guillemet, K. Bouzehouane, S. Fusil, and A. Fert, Nat. Nanotechnol. 4 , 528 (2009). 